\title{
Helical Topological Edge States in a Quadrupole Phase
}

\author{
Feng Liu, ${ }^{1}$ Hai-Yao Deng, ${ }^{2}$ and Katsunori Wakabayashi ${ }^{1}$ \\ ${ }^{1}$ Department of Nanotechnology for Sustainable Energy, School of Science and Technology, \\ Kwansei Gakuin University, Gakuen 2-1, Sanda 669-1337, Japan \\ ${ }^{2}$ School of Physics, University of Exeter, Stocker Road, EX4 4QL Exeter, United Kingdom
}

(Received 26 November 2018; published 1 March 2019)

\begin{abstract}
A topological electric quadrupole is a recently proposed concept that extends the theory of electric polarization of crystals to higher orders. Such a quadrupole phase supports topological states localized on both edges and corners. In this work, we show that in a quadrupole phase of a honeycomb lattice, topological helical edge states and pseudospin-polarized corner states appear by making use of a pseudospin degree of freedom related to point group symmetry. Furthermore, we argue that a general condition for the emergence of helical edge states in a (pseudo)spinful quadrupole phase is the existence of either mirror or time-reversal symmetry. Our results offer a way of generating topological helical edge states without spin-orbital couplings.
\end{abstract}

DOI: 10.1103/PhysRevLett.122.086804

The concept of topology in electronic materials has offered us a unique dimension to design materials with useful properties [1-3]. Especially in topological insulators (TIs), a dissipationless spin current flows along the edges of a strip in the absence of charge current [4,5]. These topological helical edge states have potential applications in low-power electronics [6]. Realization of topological helical edge states usually requires a spin-orbital coupling. How to realize topological helical edge states without spin-orbit couplings remains as a fundamental open question $[7,8]$.

The recently proposed topological electric multipoles such as dipoles and quadrupoles offer us a nice opportunity to attack this open question $[9,10]$. Topological electric multipole is a generalization of the modern theory of charge polarization to high dimensions [11-13], which introduces a new class of topological materials dubbed as high order TIs [14-18]. When a sample with finite topological electric dipole moment is terminated with an edge, topologically protected fractional charge will appear on the edge [19-22]. Analogously, a finite quadrupole upon being terminated develops both topological edge and corner states. Experiments have observed these topological corner states in various systems such as photonic, acoustic crystals and circuit arrays [23-25]. Remarkably, emergences of finite topological dipole and quadrupole do not require spin-orbital couplings [26,27].

In previous studies of topological electric multipole phase, (pseudo)spin degrees of freedom have not been paid much attention. Without (pseudo)spins, electric-multipole-induced edge states are topologically protected but not helical. These edge states suffer from dissipation during propagation. To overcome this shortcoming and gain fundamental understanding of topological electric multipoles, we introduce pseudospin degrees of freedom related to point group symmetry in a topological quadrupole phase. For concreteness, we consider a honeycomb lattice with Kekulé-like hopping textures. Based on this model, we argue that a general condition for the emergences of topological helical edge states in a (pseudo)spinful quadrupole phase is the existence of either mirror or time-reversal symmetry.

Before going into details of the honeycomb lattice model, let us introduce the topological electric multipoles such as dipoles and quadrupoles first. In crystalline systems, electric multipoles are related to the Berry connection in momentum space. For example, the dipole moment in a two-dimensional (2D) system can be expressed as

$$
P_{i}(k)=\frac{e}{|\mathcal{P}|} \sum_{n}^{N_{\text {occ }}} \int_{\mathcal{P}} \mathbf{A}^{n}(\mathbf{k}) \cdot \mathbf{n}_{i} d k^{\prime},
$$

where the summation is taken for all the occupied energy bands, $\mathbf{k}=\left(k, k^{\prime}\right)$, the integration is along a straight path $\mathcal{P}$ that connects two equivalent $\mathbf{k}$ points in momentum space. $\mathbf{n}_{i}$ is a unit vector along the $i$ direction, and $\mathbf{A}^{n}=i\left\langle u_{n \mathbf{k}}\left|\partial_{\mathbf{k}}\right| u_{n \mathbf{k}}\right\rangle$ is the Berry connection with $\left|u_{n \mathbf{k}}\right\rangle$ the periodic part of the Bloch state of the $n$th energy band. Because of gauge freedom, the dipole moment is well defined up to a lattice constant. Since there are two independent directions in a 2D system, the dipole moment of the $n$th energy band is written as $\mathbf{P}^{n}=\left(P_{i}^{n}, P_{j}^{n}\right)$ in general. The independent components of a dipole moment allow us to define a quadrupole as

$$
Q_{i j}=\sum_{n}^{N_{\text {occ }}} P_{i}^{n} P_{j}^{n} / e .
$$

Equation (2) clearly states that a corner state appears when both of $P_{i}^{n}$ and $P_{j}^{n}$ are not zero. The derivation of Eqs. (1) and (2) is given in Sec. A of the Supplemental Material [28]. 


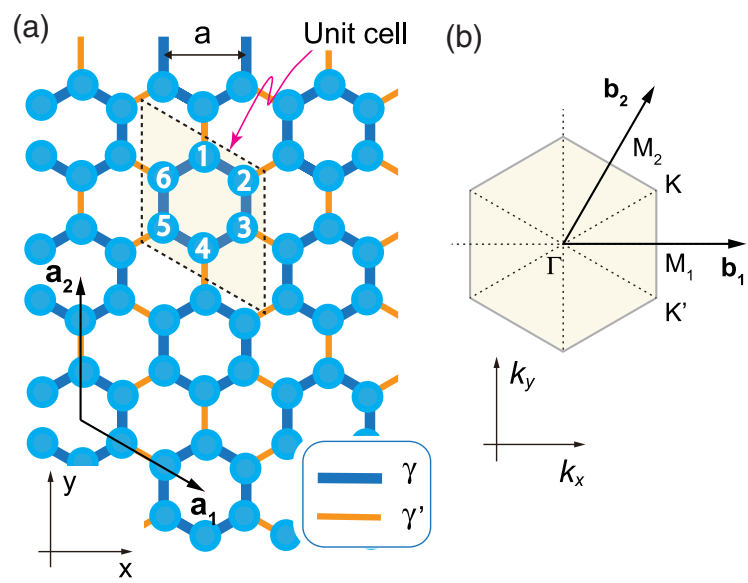

(c)
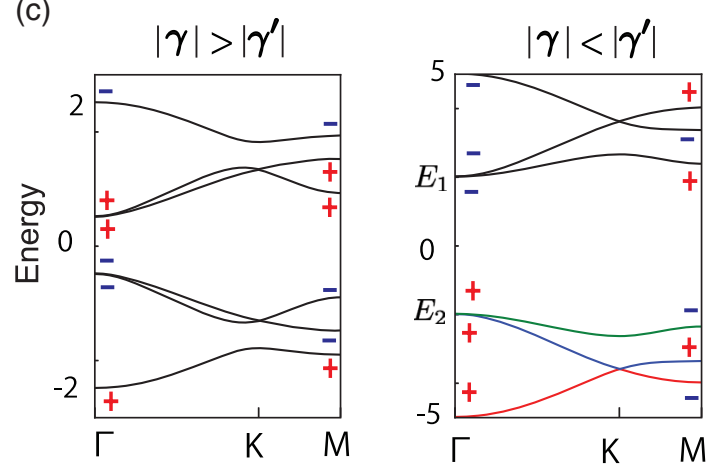

FIG. 1. (a) Schematic of the model that is characterized by two hopping parameters $\gamma$ and $\gamma^{\prime}$. Within a unit cell, there are six atomic orbitals $|j\rangle, j=1, \ldots, 6$. (b) Reciprocal lattice vectors $\mathbf{b}_{1,2}$ and the first Brillouin zone. (c) Energy bands spectrum for $|\gamma|>$ $\left|\gamma^{\prime}\right|$ and $|\gamma|<\left|\gamma^{\prime}\right|$. " \pm " indicates the eigenvalue of $\pi$ rotation of wave functions at $\Gamma$ and $M$ points. $M$ refers to either $M_{1}$ or $M_{2}$ in the 1st Brillouin zone. The first three energy bands in the case of $|\gamma|<|\gamma|^{\prime}$ are colored as red, blue, and green, respectively, for clarifying the dipole moment of each band.

The honeycomb lattice with a Kekulé-like hopping texture is displayed in Fig. 1(a) [29,30]. There are two types of hopping parameters such as intracell hopping $\gamma$ and intercell hopping $\gamma^{\prime}$ similar to the Su-Schrieffer-Heeger (SSH) model [31,32]. Resembling the SSH model, a topological dipole appears when $|\gamma|<\left|\gamma^{\prime}\right|$ [30]. Because of the $C_{6 v}$ point group symmetry that dipole moment is not zero for both $\mathbf{b}_{1}$ and $\mathbf{b}_{2}$ directions, a finite quadrupole may exist. Here $\mathbf{b}_{1}$ and $\mathbf{b}_{2}$ are the primitive lattice vectors of the reciprocal lattice, which form the 1st Brillouin zone as shown in Fig. 1(b). The energy band spectrums for $|\gamma|>$ $\left|\gamma^{\prime}\right|$ and $|\gamma|<\left|\gamma^{\prime}\right|$ are displayed in Fig. 1(c). In the case of $|\gamma|<\left|\gamma^{\prime}\right|$, band inversions happen at $\Gamma$ and $M$. A detailed energy bands' evolution by changing $\gamma / \gamma^{\prime}$ is given in Sec. B of the Supplemental Material [28].

Since numerical evaluation of $\mathbf{A}^{n}(\mathbf{k})$ produces very spiky function in momentum space due to the gauge freedom of wave functions, it is difficult to obtain the dipole moment numerically using Eq. (1). However, under a zero Berry curvature $\left(\mathcal{F}=\partial_{i} A_{j}-\partial_{j} A_{i}\right)$ [33], based on



FIG. 2. Schematic of pseudospins made up by linear combination of atomic orbitals. Pseudo spin-up and -down transform to each by either time-reversal operation or mirror reflection. Inset: combination factors indicated in color.

Eq. (1) the dipole moment can be determined by the eigenvalue of $C_{2}$ rotation at high-symmetric k points such as $[13,30]$

$$
P_{i}^{n}=\frac{1}{2}\left(q_{i}^{n} \text { modulo } 2\right), \quad(-1)^{q_{i}^{n}}=\frac{\eta_{n}\left(M_{i}\right)}{\eta_{n}(\Gamma)},
$$

where $\eta^{n}(\mathbf{k})$ is the eigenvalue of $\pi$ rotation over the $z$ axis at $\mathbf{k}$ point for the $n$th energy band. Then on the basis of the eigenvalue of $\pi$ rotation at $\Gamma$ and $M_{i}$ shown in Fig. 1(c) for $|\gamma|<\left|\gamma^{\prime}\right|$, we obtain dipole moments $e / 2,0, e / 2$ for the 1 st (red), 2nd (blue), and 3rd (green) occupied bands, respectively. Similarly, the quadrupoles are $e / 4,0$, and $e / 4$, respectively, as $P_{1}^{n}=P_{2}^{n}$ guaranteed by the $C_{6 v}$ point group symmetry. From Eqs. (1) and (2) the total dipole moment vanishes and the total quadrupole is $e / 2$. Thus, topological edge and corner states appear owing to the finite quadrupole $e / 2$ when $|\gamma|<\left|\gamma^{\prime}\right|$. In the following we show that by introducing a pseudospin degree of freedom related to $C_{6 v}$ point group symmetry, topological helical edge states and pseudospin-polarized corner states appear.

As shown in Fig. 1(c), owing to $C_{6 v}$ point group symmetry, there are two pairs of doubly degenerate states at $\Gamma$, i.e., $E_{1}$ and $E_{2}$ that may be regarded as pseudospins. For $E_{1}$ states, we call them $\left|E_{11}\right\rangle$ and $\left|E_{12}\right\rangle$, which are given by $\left|E_{11}\right\rangle,\left|E_{12}\right\rangle=\sum_{j} e^{ \pm \mathrm{i}(j-1) \rho}|j\rangle$. Similarly, $\left|E_{21}\right\rangle,\left|E_{22}\right\rangle=$ $\sum_{j} e^{ \pm \mathrm{i}(j-1) \omega}|j\rangle$. Here $|j\rangle(j=1, \ldots, 6)$ indicates six atomic orbitals in a unit cell as indexed in Fig. 1(a), $\rho=\pi / 6$ and $\omega=\pi / 3$. As $E_{i 1}$ and $E_{i 2}$ transform into each other under mirror reflection and also time reversal similar to real spins as depicted in Fig. 2, we regard them as pseudospin degrees of freedom defined as

$$
\begin{array}{rlrl}
\left|1_{\uparrow}\right\rangle & =\left|E_{11}\right\rangle, & & \left|2_{\uparrow}\right\rangle=\left|E_{21}\right\rangle, \\
\left|1_{\downarrow}\right\rangle=\left|E_{12}\right\rangle, & \left|2_{\downarrow}\right\rangle=\left|E_{22}\right\rangle .
\end{array}
$$



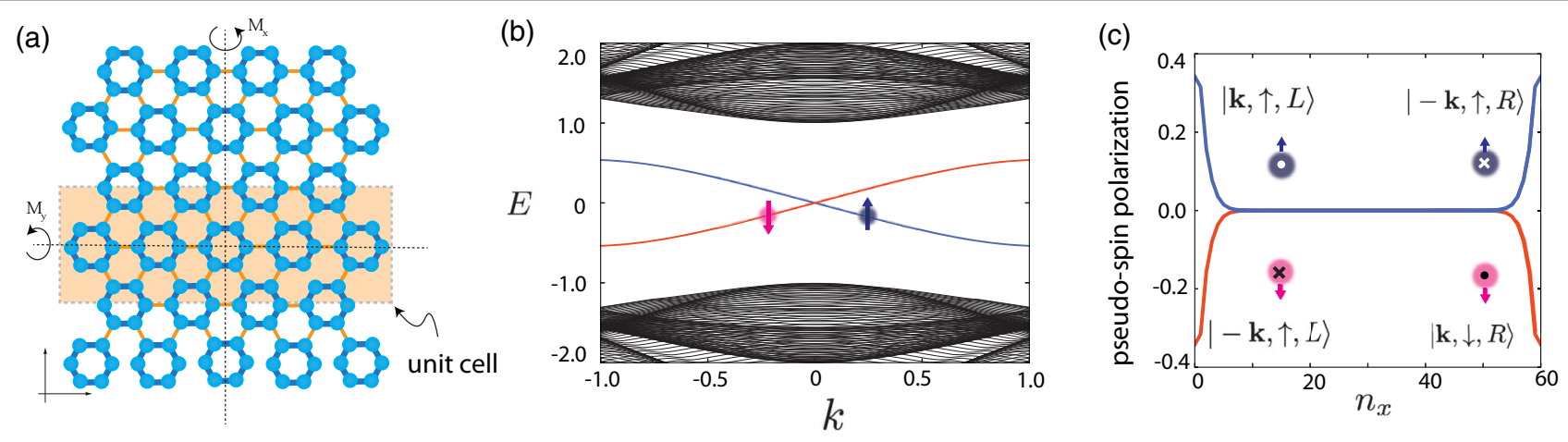

FIG. 3. (a) Schematic of a ribbon supporting topological helical edge states. The ribbon is periodic along the $y$ direction. There are two mirror planes denoted as $M_{x}$ and $M_{y}$. (b) Energy spectrum of the ribbon for $\gamma=-1.0$ and $\gamma^{\prime}=-2.0$. The wave number $k$ refers to direction $\mathbf{b}_{1}$. Within the bulk energy gap, a pair of spin-polarized bands consisting of edge states appear. (c) Helical edge states. Because of mirror symmetry (time reversal symmetry), edge states of the same energy must propagate oppositely-as indicated by the cross and dot—with opposite spin polarization on the same edge.

In our model, there is no difference between pseudospins and real spins, we simply call them spins from now on.

To demonstrate the existence of helical edge states, we consider a ribbon structure extended along the $\mathbf{a}_{2}$ direction as displayed in Fig. 3(a). By solving its corresponding Hamiltonian, we obtain the energy spectrum of the ribbon as displayed in Fig. 3(b). It is clear to see that there is a pair of edge-state energy bands appearing within the band gap in Fig. 3(b). To show that these edge states are helical, we calculate the spin polarization defined by $\mathrm{SP}=$ $\left|\left\langle\alpha_{\uparrow} \mid \psi\right\rangle\right|^{2}-\left|\left\langle\alpha_{\downarrow} \mid \psi\right\rangle\right|^{2}$, where $\alpha=1,2$ and $|\psi\rangle$ is an edge state vector. Taking $k= \pm 0.2$ and the lower branch of edge states in Fig. 3(b) as an example, we show the spinpolarization value of the edge states in Fig. 3(c). From Fig. 3(c) we see that the edge states of same $\mathbf{k}$ but opposite spins are located separately, resulting in finite spin polarization. It is noted that these helical topological edge states are also characterized by the $Z_{2}$ invariant, Fu-Kane-Mele index, which is determined by the $C_{2}$ eigenvalue of occupied wave functions at four high-symmetric $\mathbf{k}$ points such as $\Gamma, M_{1}, M_{2}$, and $M_{3}$ [34]. Because of the $C_{6 v}$ point group symmetry of the unit cell displayed in Fig. 1(a), the Fu-Kane-Mele index and quadrupole moment always coincide. A demonstration of dissipationless transport owing to the topological helical edge states is given in Sec. C of the Supplemental Material [28,35].

Besides edge states, corner states also emerge in a quadrupole phase, which is an essential signature that distinguishes the high-order TIs from conventional TIs. We should stress that the emergence of corner states does not require a global band gap of edge states. To demonstrate the existence of corner states, we consider a finite sample spanning $10 \times 10$ unit cells with open boundaries as displayed in Fig. 4(a). This sample has two types of edges and also two types of corners, i.e., zigzag and armchair. By solving its Hamiltonian, we observe eight corner states in total as there are four corners and also two spins. Four of the corner states have positive energies, and the other four have negative energies. Corner states localized at zigzag and armchair corners also have different energies. The eigenenergies of the corner states are located within the band gap between the bulk and edge states, which is $0.05 \gamma$ from edge states and $0.5 \gamma$ from bulk states, large enough to separate them from the continuum of extended Bloch states of bulk and edges. For each corner state, there is $e / 2$ amount of charge. In Sec. D of the Supplemental Material the detailed charge distribution of (a)

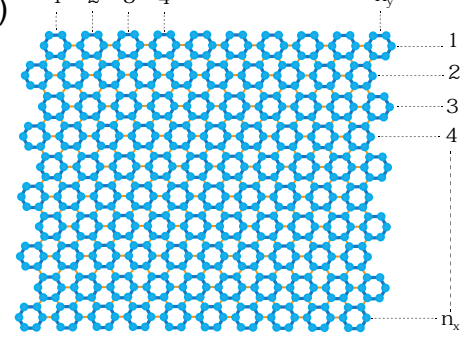

(b)

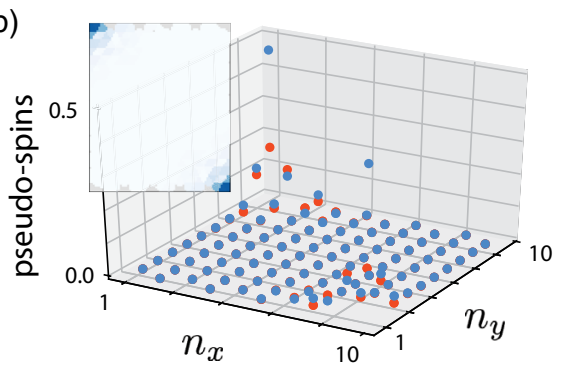

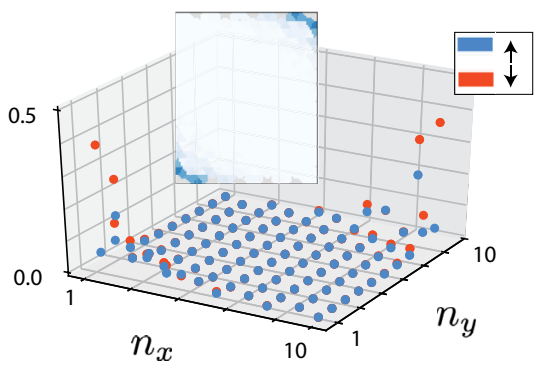

FIG. 4. (a) Schematic of a sample comprised of $10 \times 10$ unit cells supporting the corner states. We call the edge along the $x$ direction zigzag, while that along the $y$ direction armchair. (b) Spin decomposition of the corner states with positive energies, blue (red) for the spin-up (-down) component. Zigzag corner states are spin-polarized up while armchair corner states are spin-polarized down. Insets: charge density maps for the corner states. 
corner states is given [28]. As corner states have zero momentum, the spin-up and spin-down corner states are degenerate due to time-reversal symmetry. Thus, the corner states are not polarized along the $z$ direction of spins. But due to finite spin-spin coupling, they are polarized along the $x$ or $y$ direction of spins. To see this, we redefine the spins by $\left|\alpha_{\uparrow}^{\prime}\right\rangle,\left|\alpha_{\downarrow}^{\prime}\right\rangle=\left|\alpha_{\uparrow}\right\rangle \pm \mathrm{i}\left|\alpha_{\downarrow}\right\rangle$ with $\alpha=1,2$. Then taking the corner states with positive energies as an example, we calculate their spin polarization according to this definition. The result is displayed in Fig. 4(b). We see that for zigzag corners, the corner states are spin-polarized up, whereas, for armchair ones, the states are polarized down. The spin polarization of each corner state is constant regardless of the values of $\gamma$ and $\gamma^{\prime}$ as long as $\left|\gamma^{\prime} / \gamma\right|>1$, testifying to their topological nature. These spin-polarized topological corner states may work as spinful quantum dots, with potential applications in spintronics.

In the above discussions, we have shown the existence of topological helical edge states and also spin-polarized corner states in the honeycomb model. Here we try to find a general condition for emergence of helical edge states in a spinful quadrupole phase. We denote the spinful quadrupole-induced localized states on the edge $\zeta$ with momentum $\mathbf{k}$ and $\operatorname{spin} \sigma=(\uparrow, \downarrow)$ as $|\mathbf{k}, \sigma, \zeta\rangle$. Here $\zeta(=L, R)$ indicates left-side edge " $L$ " or right-side edge " $R$ " of a ribbon. To obtain spin-polarized edge states, it is required that $|\mathbf{k}, \uparrow, \zeta\rangle$ and $|\mathbf{k}, \downarrow, \zeta\rangle$ are not degenerate as shown in the honeycomb model. To fulfill this condition, we check if there is any symmetry connecting these two states. Here we consider three elementary symmetries such as time-reversal $\mathcal{T}$, mirror reflections $\mathcal{M}_{x}$ and $\mathcal{M}_{y}$, and $\pi$ rotation along the $z$ direction $R_{\pi}$. Simply we have

$$
\begin{aligned}
\mathcal{T}|\mathbf{k}, \sigma, \zeta\rangle & =|-\mathbf{k}, \bar{\sigma}, \zeta\rangle, \\
\mathcal{M}_{x}|\mathbf{k}, \sigma, \zeta\rangle & =|\mathbf{k}, \bar{\sigma}, \bar{\zeta}\rangle, \\
\mathcal{M}_{y}|\mathbf{k}, \sigma, \zeta\rangle & =|-\mathbf{k}, \bar{\sigma}, \zeta\rangle, \\
\mathcal{R}_{\pi}|\mathbf{k}, \sigma, \zeta\rangle & =|-\mathbf{k}, \sigma, \bar{\zeta}\rangle,
\end{aligned}
$$

where $\sigma, \bar{\sigma}$ have opposite values, and so as $\zeta, \bar{\zeta}$. From the above relations, it is noticed that the above symmetric operations change either two of these three "quantum numbers." Suppose that the $|\mathbf{k}, \uparrow, L\rangle$ state is $|0,0,0\rangle$ and the $|\mathbf{k}, \downarrow, L\rangle$ is $|0,1,0\rangle$, it seen that any single and combinations of these symmetric operations cannot connect the two edge states as these symmetric operations conserve the summation parity of these three quantum numbers. In other words, in a spinful quadrupole phase, the edge states are spin polarized in general, which is a quite unconventional result.

Finally, we discuss the relation of the proposed honeycomb model with conventional TIs that are supported by spin-orbital couplings. In the honeycomb model, edge and corner states are protected by finite charge polarization,
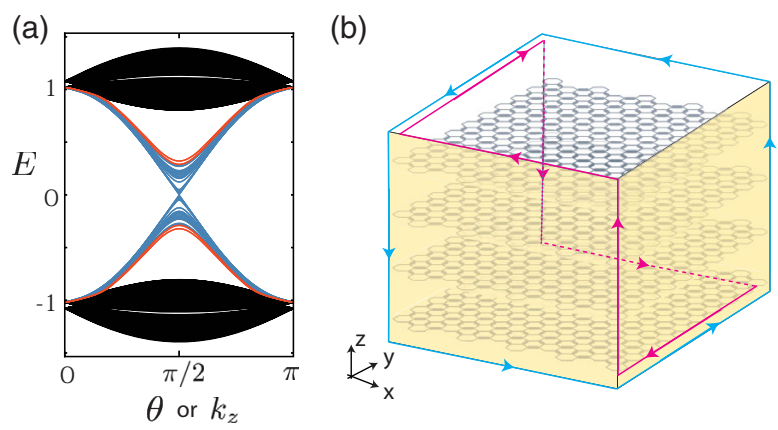

FIG. 5. (a) Pumping spectrum of a spinful quadrupole for a half period. The spectrum consists of three types of states: the bulk states (black), edge states (blue), and corner states (red). (b) By replacing $\theta$ with a quasi wave number $k_{z}$ along the third direction, a class of 3D topological insulators characterized by spin polarized hinge states - blue for spin-up and red for spin-down-emerge.

which corresponds to a winding phase of a connection defined by Bloch functions in momentum space as shown in Eq. (1). The nonzero winding phase can also be expressed as an integration of a curvature by adding one extra dimension [20]. As discussed in Ref. [36], by dimensional reduction a $2 \mathrm{D}$ TI can be mapped to a $1 \mathrm{D}$ $\mathrm{SSH}$ model. Thus, the proposed honeycomb model that is similar to the 2D SSH model corresponds to a new type of 3D TI. To see this, we investigate an adiabatic pumping process of the spinful quadrupole in the honeycomb model controlled by parameter $\theta$. Namely, we set $\left(\mu, \gamma, \gamma^{\prime}\right)=$ $(\cos \theta, 0.2, \sin \theta)$, where $\mu$ is a staggered on-site potential with opposite signs on the even- and odd-numbered atomic orbitals in a unit cell. The pumping spectrum for half period of the finite sample of Fig. 4(a) is displayed in Fig. 5(a). It is made up of three portions - bulk, edge, and corner states, which are shown as black, blue, and red in Fig. 5(a), respectively. By replacing the pumping parameter $\theta$ with a quasimomentum $k_{z}$ along the third direction, we end up with a class of 3D TIs featuring spin-polarized surface and hinge states as shown in Fig. 5(b). To realize this type of 3D TIs, one may stack the honeycomb structure with on-site potentials depending on the layer index and a small interlayer hopping.

We have discussed a spinful quadrupole phase as exemplified on a honeycomb lattice with Kekulé-like hopping texture. With neither spin-orbital couplings nor external fields, topological helical edge states closely resembling those in conventional TIs have been created plus spinpolarized corner states. By an adiabatic pumping process of spinful quadrupole, we have defined a new class of threedimensional topological insulators characterized by spinpolarized surface and hinge states. These results are expected to be useful for understanding the topological properties of crystalline systems and designing novel topological materials for low-power electronics. 
[1] K. v. Klitzing, G. Dorda, and M. Pepper, Phys. Rev. Lett. 45, 494 (1980).

[2] F. D. M. Haldane, Phys. Rev. Lett. 61, 2015 (1988).

[3] M. Z. Hasan and C. L. Kane, Rev. Mod. Phys. 82, 3045 (2010).

[4] C. L. Kane and E. J. Mele, Phys. Rev. Lett. 95, 226801 (2005).

[5] B. A. Bernevig, T. L. Hughes, and S.-C. Zhang, Science 314, 1757 (2006).

[6] M. König, H. Buhmann, L. W. Molenkamp, T. Hughes, C.-X. Liu, X.-L. Qi, and S.-C. Zhang, J. Phys. Soc. Jpn. 77, 031007 (2008).

[7] L. Fu, Phys. Rev. Lett. 106, 106802 (2011).

[8] A. Alexandradinata, C. Fang, M. J. Gilbert, and B. A. Bernevig, Phys. Rev. Lett. 113, 116403 (2014).

[9] W. A. Benalcazar, B. A. Bernevig, and T. L. Hughes, Science 357, 61 (2017).

[10] W. A. Benalcazar, B. A. Bernevig, and T. L. Hughes, Phys. Rev. B 96, 245115 (2017).

[11] J. Zak, Phys. Rev. Lett. 62, 2747 (1989).

[12] N. Marzari, A. A. Mostofi, J. R. Yates, I. Souza, and D. Vanderbilt, Rev. Mod. Phys. 84, 1419 (2012).

[13] C. Fang, M. J. Gilbert, and B. A. Bernevig, Phys. Rev. B 86, 115112 (2012).

[14] Z. Song, Z. Fang, and C. Fang, Phys. Rev. Lett. 119, 246402 (2017).

[15] M. Geier, L. Trifunovic, M. Hoskam, and P. W. Brouwer, Phys. Rev. B 97, 205135 (2018).

[16] X. Zhu, Phys. Rev. B 97, 205134 (2018).

[17] T. Fukui and Y. Hatsugai, Phys. Rev. B 98, 035147 (2018).

[18] B.-Y. Xie, H.-F. Wang, H.-X. Wang, X.-Y. Zhu, J.-H. Jiang, M.-H. Lu, and Y.-F. Chen, Phys. Rev. B 98, 205147 (2018).

[19] R. D. King-Smith and D. Vanderbilt, Phys. Rev. B 47, 1651 (1993).

[20] R. Resta, Rev. Mod. Phys. 66, 899 (1994).

[21] Y. Zhou, K. M. Rabe, and D. Vanderbilt, Phys. Rev. B 92, 041102 (2015).
[22] Y. Ota, D. Takamiya, R. Ohta, H. Takagi, N. Kumagai, S. Iwamoto, and Y. Arakawa, Appl. Phys. Lett. 112, 093101 (2018).

[23] C. W. Peterson, W. A. Benalcazar, T. L. Hughes, and G. Bahl, Nature (London) 555, 346 (2018).

[24] M. Serra-Garcia, V. Peri, R. Süsstrunk, O. R. Bilal, T. Larsen, L. G. Villanueva, and S. D. Huber, Nature (London) 555, 342 (2018).

[25] S. Imhof, C. Berger, F. Bayer, J. Brehm, L. W. Molenkamp, T. Kiessling, F. Schindler, C. H. Lee, M. Greiter, T. Neupert, and R. Thomale, Nat. Phys. 14, 925 (2018).

[26] F. Liu and K. Wakabayashi, Phys. Rev. Lett. 118, 076803 (2017).

[27] F. Liu, H.-Y. Deng, and K. Wakabayashi, Phys. Rev. B 97, 035442 (2018).

[28] See Supplemental Material at http://link.aps.org/ supplemental/10.1103/PhysRevLett.122.086804 for more details.

[29] T. Kariyado and X. Hu, Sci. Rep. 7, 16515 (2017).

[30] F. Liu, M. Yamamoto, and K. Wakabayashi, J. Phys. Soc. Jpn. 86, 123707 (2017).

[31] P. Delplace, D. Ullmo, and G. Montambaux, Phys. Rev. B 84, 195452 (2011).

[32] S. Sorella, K. Seki, O. O. Brovko, T. Shirakawa, S. Miyakoshi, S. Yunoki, and E. Tosatti, Phys. Rev. Lett. 121, 066402 (2018).

[33] In this honeycomb model, the zero Berry curvature condition can be fulfilled by adding tiny unsymmetric onsite potentials, which have negligible effects on the properties we are interested in. For example, one can put $(0,0.01,0.02,0$, $0.01,0.02$ ) onsite potentials for 1 st to 6 th atomic orbitals as indexed in Fig. 1(a). Then because of simultaneous presences of time-reversal symmetry and inverison symmetry, the Berry curvature vanishes everywhere in momentum space.

[34] L. Fu and C. L. Kane, Phys. Rev. B 76, 045302 (2007).

[35] C. W. Groth, M. Wimmer, A. R. Akhmerov, and X. Waintal, New J. Phys. 16, 063065 (2014).

[36] X.-L. Qi, T. L. Hughes, and S.-C. Zhang, Phys. Rev. B 78, 195424 (2008) 